\title{
Chirality in Geometric Algebra
}

\author{
Michel Petitjean 1,2 (D) \\ 1 BFA, CNRS UMR 8251, INSERM ERL U1133, Université de Paris, 75013 Paris, France; \\ petitjean.chiral@gmail.com or michel.petitjean@univ-paris-diderot.fr \\ 2 E-pôle de Génoinformatique, CNRS UMR 7592, Institut Jacques Monod, 75013 Paris, France
}

check for

updates

Citation: Petitjean, M. Chirality in Geometric Algebra. Mathematics 2021, 9, 1521. https://doi.org/ $10.3390 /$ math 9131521

Academic Editor: Gabriel Eduard Vilcu

Received: 11 May 2021

Accepted: 26 June 2021

Published: 29 June 2021

Publisher's Note: MDPI stays neutral with regard to jurisdictional claims in published maps and institutional affiliations.

Copyright: (c) 2021 by the author. Licensee MDPI, Basel, Switzerland. This article is an open access article distributed under the terms and conditions of the Creative Commons Attribution (CC BY) license (https:/ / creativecommons.org/licenses/by/ $4.0 /)$.

\begin{abstract}
We define chirality in the context of chiral algebra. We show that it coincides with the more general chirality definition that appears in the literature, which does not require the existence of a quadratic space. Neither matrix representation of the orthogonal group nor complex numbers are used.
\end{abstract}

Keywords: chirality; symmetry; reflections; geometric algebra

MSC: 11E88; 15A66

\section{Introduction to Symmetry and Chirality}

Although symmetry and chirality have been known about for millennia, in the arts, architecture, and elsewhere they have recently received a mathematical definition establishing consensual terminology. It is necessary to mention several important contributions.

According to science historian Joseph Gal [1], Pasteur discovered molecular chirality in 1848 , but used the term dissymmetry (a detailed history is given in Gal's paper). However, dissymmetry should not be confused with asymmetry, which means a lack of both direct and indirect symmetry.

The terms chiral and chirality were introduced later, in 1894, by Lord Kelvin [2,3], and dissymmetry is now obsolete. Quoting Lord Kelvin: I call any geometrical figure, or group of points, chiral, and say that it has chirality if its image in a plane mirror, ideally realized, cannot be brought to coincide with itself. This definition is clear, but it is not mathematical, in a modern sense. It was thought for Euclidean spaces rather than for the spacetime, but it is still widely used for teaching.

The term chirality was reintroduced in 1946 by Eddington [4], who wrote that the term was introduced by Kelvin. He also used the terms right-handed and left-handed, and that seems to have been at the basis of the use of these terms in quantum field theory and related fields.

Symmetry has been studied extensively in geometric algebra $[5,6]$. Chirality is intimately related to symmetry. A mathematical definition of symmetry can be found in [7]. It works in many cases, such as for geometric figures (with or without colors, as encountered in arts), for functions, probability distributions, graphs, matrices, strings, etc. This definition assumed the existence of a metric, but in fact, none of the axioms defining a true metric was necessary to define isometries. Therefore, it works in geometric algebra, for which intervals are preserved, rather than distances. In particular, it permits definition of what can be qualified as symmetric or not, as recalled in Definitions 1 and 2. Then, Definition 4 of chirality works in geometric algebra.

Definition 1. An object is a function with its input argument in a metric space [7].

The space of the returned values should be defined by the user. By extension, we retain here the term object when the metric is not a true one, as it happens in geometric algebra. 
Definition 1 is pertinent in the case of the spacetime, where a physical quantity, called a field, receives a value at each point of the spacetime. Such a field should not be confused with a mathematical field, which is an algebraic structure. For example, in physics, a scalar field is a field returning a scalar value, while in mathematics, a field of scalars is a set over which is defined a vector space. Then, we retrieve the well-known classical definitions.

Definition 2. An object is symmetric when it is invariant under an isometry which is not the identity [7].

In physics, Definition 2 corresponds to global symmetry. Local symmetry and gauge symmetry are other concepts commonly encountered in physics [8]. Nevertheless, supplying them a mathematical definition working outside physics does not fall in the scope of this paper.

In the Euclidean case, rotations are classified as direct isometries, while a reflection and the compositions of rotations with an odd number of reflections are classified as indirect isometries.

The definition of direct and indirect symmetries which is retained here in Definition 3, is not the classical one related to orientation preserving operators. It is a more general one [9], which recovers the classical one in the case of Euclidean spaces. It does not require the existence of a vector space. We recall it below.

Definition 3. (a) An isometry is direct when it can be expressed as a product of squared isometries, a squared isometry being the composition of an isometry with itself.

(b) An isometry which is not direct is an indirect isometry.

Then, we retrieve the classical definition of chirality.

Definition 4. An object is achiral when it is invariant under an indirect isometry. If no indirect isometry leaves an object invariant, it is chiral.

In this paper, we show that Definition 3 works in geometric algebra, and that the isometries of the orthogonal group are classified accordingly in Section 4.5. The main result is given in Theorem 13.

\section{Basic Concepts}

The basics of geometric algebra can be found in [10-12]. Here we consider a vector space $V$ over a field $F$. In all what follows, we set $F=\mathbb{R}$. The dimension $n$ of $V$ is finite. The space $V$ is equipped with a non-degenerate quadratic form $Q$, which is not required to be positive definite. The signature of $Q$ is $(p, q, 0)$, with $p+q=n$. In this section, unless otherwise stated, $u, v, x$ and $y$ are vectors of $V$.

The quadratic space $(V, Q)$ is endowed with the symmetric bilinear form:

$$
\beta(u, v)=\frac{1}{2}[Q(u+v)-Q(u)-Q(v)]
$$

In the three next subsections, we just list known facts and results that are needed further in the text.

\subsection{The Geometric Product}

- For any vector $u, Q(u)=\beta(u, u)$.

- The inner product between two vectors $u$ and $v$ is: $u \cdot v=\beta(u, v)$.

- The subspace of isotropic vectors can be non-empty.

It happens for the Minkowski space, where isotropic vectors are called lightlike.

- The interval between two vectors $u$ and $v$ is: $\mathcal{S}_{u, v}^{2}=Q(u-v)=(u-v) \cdot(u-v)$. The interval $\mathcal{S}_{u, v}$ is defined by its square, but this square can be negative. 
- The exterior product (or wedge product) of $u$ and $v$ is denoted $u \wedge v$.

- The geometric product of $u$ and $v$ is: $u v=u \cdot v+u \wedge v$.

- The geometric product can be extended to an arbitrary number of vectors.

It is associative. It is distributive over the addition of the algebra.

- The symmetric part $u \cdot v=v \cdot u$ of the geometric product $u v$ is a scalar.

- Its antisymmetric part $u \wedge v=-v \wedge u$, is a bivector.

\subsection{Invertible Vectors}

- For any vector $u, u \wedge u=0$, and $u u=u \cdot u$.

- For any vectors $u$ and $v, u^{2} v^{2}=(u \cdot v)^{2}-(u \wedge v)^{2}$.

- Two vectors $u$ and $v$ are said to be orthogonal when $u \cdot v=0$.

- A unit vector $u$ is such that $u \cdot u= \pm 1$.

- The inverse of a vector $v, v \cdot v \neq 0$ is: $v^{-1}=\frac{1}{v \cdot v} v$. It is such that $v v^{-1}=v^{-1} v=1$. Isotropic vectors cannot be scaled to unit and cannot be inverted.

\subsection{Projections and Reflections}

- The projection $x_{\| v}$ of a vector $x$ onto an invertible vector $v$ is: $x_{\| v}=(x \cdot v) v^{-1}$.

- The rejection part of $x$ is: $x_{\perp v}=x-x_{\| v}$. Thus, $x_{\perp v}=(x \wedge v) v^{-1}$.

- $x_{\perp v}$ and $v$ are orthogonal because $x_{\perp v} \cdot v=0 ; x \cdot x=x_{\| v} \cdot x_{\| v}+x_{\perp v} \cdot x_{\perp v}$.

- The reflection $\sigma_{v}$ is an operator which transforms a vector $x$ into its reflected image through the hyperplane orthogonal to $v: \sigma_{v}(x)=-x_{\| v}+x_{\perp v}$.

- It is deduced the following expression of $\sigma_{v}(x)$ :

$$
\sigma_{v}(x)=-v x v^{-1}
$$

In the literature, the opposite of the right side of Equation (2) is sometimes called the sandwiching product.

- A reflection is linear, involutive, and bijective.

- The reflection $\sigma_{v}$ is invariant when $v$ is multiplied by a non-null constant $\lambda: \sigma_{v}=\sigma_{\lambda v}$.

Therefore, Equation (2) can be expressed with the unit vector $u=\frac{1}{|v \cdot v|^{1 / 2}} v$, which is such that $u \cdot u=\operatorname{sgn}(v \cdot v)= \pm 1$.

$$
\sigma_{u}(x)=-\epsilon_{u} u x u, \quad \epsilon_{u}=\operatorname{sgn}(u \cdot u)
$$

Lemma 1. Given an invertible vector $v$, the reflection $\sigma_{v}$ preserves intervals.

Proof. Consider any vector $x ; \sigma_{v}(x) \cdot \sigma_{v}(x)=v x v^{-1} \cdot v x v^{-1}$, i.e., $\sigma_{v}(x) \cdot \sigma_{v}(x)=v x v^{-1} v x v^{-1}$. Then, $x x=x \cdot x$, and it comes that $\sigma_{v}(x) \cdot \sigma_{v}(x)=x \cdot x$. Similarly, for any two vectors $x$ and $y, \sigma_{v}(x-y) \cdot \sigma_{v}(x-y)=(x-y) \cdot(x-y)$.

Lemma 2. Given an invertible vector $v$, the reflection $\sigma_{v}$ preserves the inner product.

Proof. Consider any two vectors $x$ and $y$. Using Equation (1), express $\sigma_{v}(x) \cdot \sigma_{v}(y)$ as a linear combination of squared vectors, then apply Lemma 1.

Theorem 1. The composition of any number of reflections is a bijection of $V$ onto $V$ preserving the inner product.

Proof. Apply Lemma 2 and remember that a reflection is a bijection of $V$ onto $V$.

According to [10], a product of vectors is a called a versor, and it is called a $k$-versor when it is factorized into a product of $k$ vectors. A scalar is a 0 -versor. When a versor $S$ is a product of $k$ vectors, the product expressed in reverse order is denoted $S^{\dagger}$. An even 
versor $S$ which is such that $S^{\dagger} S=+1$ is called a rotor. A rotation $R$ is a product of an even number of reflections which is so that $R(x)=S^{\dagger} x S$, where $S$ is a rotor.

Reflections and compositions of reflections are operators preserving intervals. It is why we call these operators isometries, even if the quadratic space is not a true metric space. Reflections generate a group by their composition, called the real orthogonal group $O(p, q ; \mathbb{R})$, denoted here $O(p, q)$, with $p+q=n$. When $Q$ is positive definite, $q=0$, and $O(p, q)$ is denoted $O(n)$. Translations are also called isometries because, visibly, they preserve intervals. Translations are affine transformations, they are not members of $O(p, q)$. However, a modern treatment of translations exists in geometric algebra [5,6]. The Euclidean group is a semidirect product of $O(n)$ and the group of translations. According to Cartan-Dieudonné theorem, any element of the orthogonal group can be generated by at most $n$ reflections: see [13], or Theorem 3.20 in [14].

Some other classical results are given in the next section. In all subsequent sections, we neglect translations. Thus, for convenience and by abuse of language, we use the word isometry as being synonym of a member of the orthogonal group. As for the geometric product, we do not use any symbol to denote products or compositions of isometries. That will raise no ambiguity because it will be clear from the context.

\section{Basis Vectors, Commutation, Anticommutation and Pseudoscalars}

\subsection{Basis Vectors}

The bilinear form $\beta$ is defined from the non-degenerate quadratic form $Q$ in Equation (1), which is always expressible with a $n$ by $n$ nonsingular square matrix $B$. This latter can be considered to be symmetric, without loss of generality ( $Q$ is invariant when $B$ is replaced by its transposed $B^{t}$ or by $\left.\left(B+B^{t}\right) / 2\right)$. The matrix $B$ represents a linear automorphism $\mathcal{B}$ of $V$ onto $V$. This latter has $n$ real eigenvalues and $n$ eigenvectors. For convenience, we assume that these $n$ eigenvalues are ordered in non-increasing order, so that the $p$ first eigenvalues are positive and the $q=n-p$ last eigenvalues are negative.

The quadratic space $(V, Q)$ has $n$ basis vectors $e_{i}, 1 \leq i \leq n$. These latter can be chosen as the eigenvectors associated with the eigenvalues of the linear automorphism $\mathcal{B}$. It follows that $e_{i} \cdot e_{j}=\beta\left(e_{i}, e_{j}\right)=0$ when $i \neq j$. Then, the $n$ eigenvectors are normalized: $e_{i} \cdot e_{i}=+1$ for $1 \leq i \leq p$, and $e_{i} \cdot e_{i}=-1$ for $(p+1) \leq i \leq n$.

\subsection{Commutation and Anticommutation}

Theorem 2. The product of any two vectors $u$ and $v$ cannot commute when $(u \cdot u)(v \cdot v)<0$. The product of two invertible vectors commute if and only if they are colinear.

Proof. Let $u$ and $v$ be two vectors of $V$. If $u$ and $v$ are colinear, $u v=v u$.

Conversely, we assume that $v u=u v$. Thus, $u \wedge v=0, u v=u \cdot v$, and $u v v u=$ $(u \cdot u)(v \cdot v)=(u \cdot v)^{2}$.

It follows that the quantity $(u \cdot u)(v \cdot v)$ cannot be negative.

We assume that $u$ and $v$ are invertible and that $v u=u v$. Then, $v=u v u^{-1}$, i.e., $v=u \frac{u \cdot v}{u \cdot u}$.

Corollary 1. The reflections $\sigma_{u}$ and $\sigma_{v}$, respectively along the invertible vectors $u$ and $v$, are equal if and only $u$ and $v$ are colinear.

Proof. Obviously, the colinearity of $u$ and $v$ implies that $\sigma_{u}=\sigma_{v}$.

Conversely, if we assume that $\sigma_{u}=\sigma_{v}$, we have both $\sigma_{u}(v)=-u v u^{-1}$ and $\sigma_{v}(v)=-v$, from which $u v u^{-1}=v$, i.e., $u v=v u=u \cdot v$. Apply Theorem 2 .

Any two distinct basis vectors $e_{i}$ and $e_{j}$ are orthogonal. We deduce that $e_{i} e_{j}=-e_{j} e_{i}$. This is the anticommutation rule, a cornerstone in the theory of Clifford algebras.

Theorem 3. The product of two distinct reflections $\sigma_{u}$ and $\sigma_{v}$, respectively along the invertible vectors $u$ and $v$, is commutative if and only if $u$ and $v$ are othogonal. If so, it is involutive. 
Proof. We define $\epsilon_{u}=\operatorname{sgn}(u \cdot u)$ and $\epsilon_{v}=\operatorname{sgn}(v \cdot v)$, and we assume that $u$ and $v$ are unit vectors, without loss of generality. From Equation (3), we obtain that $v u x u v=u v x v u$ for any vector $x$. We deduce that $4(u \cdot v) x(u \wedge v)=(u v+v u) x(u v-v u)=u v x u v-v u x v u$. Setting $x=u$, we obtain $(u \cdot v) u(u \wedge v)=0$. Thus, either $u \cdot v=0$ or $u(u \wedge v)=0$. However, in this latter situation, we would have $u(u v)=u(u \cdot v)$, meaning that $u$ and $v$ are colinear, and that $\sigma_{u}=\sigma_{v}$ due to Corollary 1 .

Conversely, if $u \cdot v=0, u v=-v u$, vuxuv $=u v x v u$, and $\sigma_{v} \sigma_{u}=\sigma_{u} \sigma_{v}$.

If $\sigma_{v} \sigma_{u}$ is commutative, it is involutive because $\left(\sigma_{v} \sigma_{u}\right)^{2}=\left(\sigma_{v} \sigma_{u}\right)\left(\sigma_{u} \sigma_{v}\right)$.

\subsection{Pseudoscalars}

Lemma 3. The wedge product of $m$ vectors of $V, v_{1} \wedge \ldots \wedge v_{m}$, is null when one of these vectors is linearly dependent on the other ones.

Proof. Express each of the $m$ vectors with the basis vectors and develop the product. Each term contains twice one of the basis vectors, so, all terms are null.

Definition 5. We define the following $n$-blade, of grade $n$ :

$$
\omega=e_{1} e_{2} \ldots e_{n} \text {, i.e., } \omega=e_{1} \wedge e_{2} \wedge \ldots \wedge e_{n} \text {. }
$$

Theorem 4. The following properties of $\omega$ stand:

(a) For any $v \in V, \omega \wedge v=0, \omega^{\dagger} \wedge v=0$; $w v=(-1)^{n-1} v w, w^{\dagger} v=(-1)^{n-1} v w^{\dagger}$.

(b) When $n$ is odd, $\omega$ commutes with any multivector, and when $n$ is even, $\omega$ commutes with even grade multivectors and it anticommutes with odd grade multivectors. Same conclusions for $\omega^{\dagger}$. $(-1)^{q}$.

(c) $\omega^{2}=(-1)^{q+\frac{n(n-1)}{2}} ;\left(\omega^{\dagger}\right)^{2}=(-1)^{q+\frac{n(n-1)}{2}} ; \omega^{\dagger}=(-1)^{\frac{n(n-1)}{2}} \omega ; \omega^{\dagger} \omega=\omega \omega^{\dagger}=$

(d) $\omega$ and $\omega^{\dagger}$ are pseudoscalars.

Proof. (a) $v$ is linearly dependent on the $n$ basis vectors: apply Lemma 3.

(a)-(c) Apply the anticommutation rule; $\omega e_{i}=(-1)^{n-1} e_{i} \omega$, and $\omega^{\dagger} e_{i}=(-1)^{n-1} e_{i} \omega^{\dagger}$, $1 \leq i \leq n$.

(d) $\omega$ is a pseudoscalar, but not a scalar, because its sign changes under a sign change of anyone of the basis vectors. Similarly, $\omega^{\dagger}$ is a pseudoscalar.

\section{Direct and Indirect Isometries}

Here, we retain Definition 3 as the general definition of direct and indirect isometries (see Section 1). The subset of $O(p, q)$ containing the direct isometries is denoted $O^{D}(p, q)$. It is not empty because it contains the identity. The subset of $O(p, q)$ containing the indirect isometries is denoted $O^{I}(p, q)$. The members of the orthogonal group can be classified as direct or indirect, as shown further.

\subsection{Case of a Single Reflection}

Theorem 5. A single reflection cannot be decomposed into an even number of reflections.

Proof. The proof is immediate in the case of Euclidean spaces, but it is recalled that the meaning of indirect isometries is the one of Definition 3. We consider a reflection $\sigma_{u}$ along an invertible vector $u$. We assume that $\sigma_{u}$ is a product of $2 m$ reflections, $m \geq 1$.

Let $\sigma_{v_{i}}$ be these reflections, $1 \leq i \leq 2 m$, each $\sigma_{v_{i}}$ being a reflection along the invertible vector $v_{i}$.

The image of each basis vector $e_{i}, 1 \leq i \leq n$, is: $\sigma_{u}\left(e_{i}\right)=(-1)^{2 m}\left(v_{2 m} \ldots v_{1}\right) e_{i}$ $\left(v_{1}^{-1} \ldots v_{2 m}^{-1}\right)$.

Then, the product $\Pi$ of the $n$ images is: $\Pi=\sigma_{u}\left(e_{1}\right) \ldots \sigma_{u}\left(e_{n}\right)=\left(v_{2 m} \ldots v_{1}\right) \omega$ $\left(v_{1}^{-1} \ldots v_{2 m}^{-1}\right)$, where $\omega$ is the pseudoscalar introduced in Section 3.3. Discarding if $n$ is even or odd, from Theorem $4 \mathrm{~b}, \omega$ commutes with any even grade multivector, and thus it commutes with any product of an even number of vectors because such product is a 
linear combination of even grade multivectors. Therefore, the product of these $n$ images is $\Pi=\omega$.

However, the product of the $n$ images should be: $\Pi=(-1)^{n}\left(u e_{1} u^{-1}\right) \ldots\left(u e_{n} u^{-1}\right)$, i.e., $\Pi=(-1)^{n}\left(u \omega u^{-1}\right)$. From Theorem $4 \mathrm{~b}, \omega$ commutes with $u$ when $n$ is odd, and $\omega$ anticommutes with $u$ when $n$ is even. In both cases, $\Pi=-\omega$, a contradiction (the pseudoscalar $\omega$ is not null because $\omega^{4}=1$ ).

Corollary 2. A single reflection is an indirect isometry.

Proof. A product of squared isometries is the product of an even number of isometries. Then, apply Theorem 5.

Corollary 3. The set $O^{I}(p, q)$ is not empty: it contains at least the single reflections.

When $n=1$, all vectors are colinear, $O^{I}(1,0)$ and $O^{I}(0,1)$ contains only one reflection each.

\subsection{Square Root of a Rotation}

Lemma 4. Let $u$ and $v$ be two unit vectors of $V$, so that $u \cdot u=v \cdot v$. Either $u+v$ or $u-v$ is invertible. Both can be invertible.

Proof. We define $\epsilon_{u}=u \cdot u$ and $\epsilon_{v}=v \cdot v$. We assume that $\epsilon_{u}=\epsilon_{v}$ and we assume that both $(u+v) \cdot(u+v)=0$ and $(u-v) \cdot(u-v)=0$. Then, $\epsilon_{u}+\epsilon_{v}+2 u \cdot v=0$ and $\epsilon_{u}+\epsilon_{v}-2 u \cdot v=0$. By summation, $\epsilon_{u}+\epsilon_{v}=0$, a contradiction.

Lemma 5. Let $\sigma_{u}$ and $\sigma_{v}$ be the reflections respectively associated with the invertible unit vectors $u$ and $v$. When $u \cdot u$ and $v \cdot v$ have the same sign:

- (a) If $(u+v) \cdot(u+v) \neq 0$, the operators $R_{1}=\sigma_{(u+v)} \sigma_{u}$ and $R_{2}=\sigma_{v} \sigma_{(u+v)}$ are equal.

- (b) If $(u-v) \cdot(u-v) \neq 0$, the operators $R_{3}=\sigma_{(u-v)} \sigma_{u}$ and $R_{4}=\sigma_{v} \sigma_{(u-v)}$ are equal.

Proof. We define $\epsilon_{u}=\operatorname{sgn}(u \cdot u)$ and $\epsilon_{v}=\operatorname{sgn}(v \cdot v)$.

- (a): $(u+v) \cdot(u+v) \neq 0$. The reflected images $x_{1}$ of a vector $x$ by $R_{1}$ and $x_{2}$ by $R_{2}$ are: $x_{1}=\frac{\epsilon_{u}}{(u+v) \cdot(u+v)}(u+v) u x u(u+v)$, and $x_{2}=\frac{\epsilon_{v}}{(u+v) \cdot(u+v)} v(u+v) x(u+v) v$.

$[(u+v) \cdot(u+v)]\left(x_{1}-x_{2}\right)=(u+v) u x u(u+v)-v(u+v) x(u+v) v$.

$[(u+v) \cdot(u+v)]\left(x_{1}-x_{2}\right)=\left(\epsilon_{u}-\epsilon_{v}\right)(x+v u x u v)$, then $R_{1}(x)=R_{2}(x)$ when $\epsilon_{u}=\epsilon_{v}$.

- (b): $(u-v) \cdot(u-v) \neq 0$. Replacing $u+v$ by $u-v$, in case (a), we obtain:

$[(u-v) \cdot(u-v)]\left(x_{1}-x_{2}\right)=\left(\epsilon_{u}-\epsilon_{v}\right)(x+v u x u v)$, then $R_{3}(x)=R_{4}(x)$ when $\epsilon_{u}=\epsilon_{v}$.

Theorem 6. Let $\sigma_{v} \sigma_{u}$ be the composition of the reflections $\sigma_{u}$ and $\sigma_{v}$, respectively associated with the unit vectors $u$ and $v$. When $\operatorname{sgn}(u \cdot u)=\operatorname{sgn}(v \cdot v), \sigma_{v} \sigma_{u}$ is a rotation, and it can be written as the square of an isometry. It is a direct isometry.

Proof. $\left[\sigma_{v} \sigma_{u}\right](x)=\epsilon_{u} \epsilon_{v} v u x u v$. It is assumed that $\epsilon_{u}=\operatorname{sgn}(u \cdot u)$ and $\epsilon_{v}=\operatorname{sgn}(v \cdot v)$ are equal.

(a) We assume that $(u+v) \cdot(u+v) \neq 0$.

From Lemma $5 \mathrm{a}, R_{1}=\sigma_{(u+v)} \sigma_{u}$ and $R_{2}=\sigma_{v} \sigma_{(u+v)}$ are equal, thus $R_{2} R_{1}$ is a square.

$$
\left[R_{2} R_{1}\right](x)=\frac{\epsilon_{u} \epsilon_{v}}{[(u+v) \cdot(u+v)]^{2}} v(u+v)[(u+v) u x u(u+v)](u+v) v=\epsilon_{u} \epsilon_{v} v u x u v .
$$

Thus, for any vector $x,\left[\sigma_{v} \sigma_{u}\right](x)=\left[R_{2} R_{1}\right](x)$, therefore $\sigma_{v} \sigma_{u}$ is a square.

(b) We assume that $(u-v) \cdot(u-v) \neq 0$.

The proof is the same as in case (a), using Lemma $5 \mathrm{~b}$ rather than Lemma $5 \mathrm{a}$ : $R_{3}=\sigma_{(u-v)} \sigma_{u}$ and $R_{4}=\sigma_{v} \sigma_{(u-v)}$ are equal, $R_{3} R_{4}$ is a square, and $\sigma_{v} \sigma_{u}$ is a square.

From Lemma 4, necessarily either case (a) or case (b) occurs, therefore the rotation $\sigma_{v} \sigma_{u}$ is a square. According to Definition 3, it is a direct isometry. 
In the Euclidean case, Theorem 6 means that a rotation in the plane containing $u$ and $v$ is the product of two equal rotations of half the angle defined by $u$ and $v$. Since the sense of neither $u$ nor $v$ is meaningful, two supplementary angles are considered.

Corollary 4. A product of rotations is a rotation, and it is a direct isometry.

Proof. Apply Theorem 6, or see this corollary as a consequence of Theorem 1 in [9]: a product of direct isometries is a direct isometry.

In the case of $\mathbb{R}^{1,3}$, which is used to modelize the spacetime in special relativity, the vectors with a positive square are called timelike, and the vectors with a negative square are called spacelike. and the vectors with a null square are called lightlike. In Theorem 6, the case where $u \in V^{-}$and $v \in V^{-}$correspond to spatial rotations. The case where the rotation is in a plane defined by one timelike vector and one spacelike vector is a boost, and corresponds to a hyperbolic rotation [15]. Other cases targeted by Theorem 6 correspond to compositions of rotations and boosts. Thus, boosts are direct isometries, as mentioned in [16].

\subsection{Full Reflection and Reversal Operators}

Definition 6. We denote by $\sigma_{e_{i}}$ the reflection along the basis vector $e_{i}, 1 \leq i \leq n$, and we define the full reflection as the product: $\sigma_{\omega}=\sigma_{e_{n}} \sigma_{e_{n-1}} \ldots \sigma_{e_{1}}$.

Theorem 7. (a) The full reflection is an involution which transforms any vector of $V$ into its opposite. (b) It commutes with any isometry of $O(p, q)$.

Proof. (a) For any $x \in V, \sigma_{\omega}(x)=(-1)^{n} \omega^{\dagger} x \omega(-1)^{q}$, where $\omega$ is the pseudoscalar defined in Section 3.3. Applying Theorem $4 \mathrm{~b}, \mathrm{c}$, it comes that $\sigma_{\omega}(x)=-x$. Thus, $\sigma_{\omega}$ is an involution.

(b) Let $\sigma_{u}(x)=-u x u \epsilon_{u}$ be the image of $x$ by the reflection along a unit vector $u$, with $\epsilon_{u}=u^{2}$.

We have $\sigma_{\omega}(x) \sigma_{u}(x)=\sigma_{u}(x) \sigma_{\omega}(x)$ because $w^{\dagger}(u x u) w=u\left(w^{\dagger} x w\right) u$ (see Theorem $4 \mathrm{~b}$ ). Then, any isometry of $O(p, q)$ being a product of reflections, it commutes with $\sigma_{\omega}$.

Theorem 8. When $n$ is odd, $\sigma_{\omega}$ is an indirect isometry.

Proof. The proof is immediate in the case of Euclidean spaces, but it is recalled that the meaning of indirect isometries is the one of Definition 3. We assume that $\sigma_{\omega}$ is a product of $2 m$ reflections, $m \geq 1$. Let $\sigma_{v_{i}}$ be these reflections, $1 \leq i \leq 2 m$, each $\sigma_{v_{i}}$ being a reflection along the invertible vector $v_{i}$. The image of each basis vector $e_{i}, 1 \leq i \leq n$, is: $\sigma_{\omega}\left(e_{i}\right)=(-1)^{2 m}\left(v_{2 m} \ldots v_{1}\right) e_{i}\left(v_{1}^{-1} \ldots v_{2 m}^{-1}\right)$. Then, the product $\Pi$ of the $n$ images is: $\Pi=\sigma_{\omega}\left(e_{1}\right) \ldots \sigma_{\omega}\left(e_{n}\right)=\left(v_{2 m} \ldots v_{1}\right) \omega\left(v_{1}^{-1} \ldots v_{2 m}^{-1}\right)$. Discarding if $n$ is even or odd, from Theorem $4 \mathrm{~b}, \omega$ commutes with any even grade multivector, and thus it commutes with any product of an even number of vectors because such product is a linear combination of even grade multivectors. Therefore, the product of these $n$ images is $\Pi=\omega$. However, this product should be: $\Pi=\left(-e_{1}\right) \ldots\left(-e_{n}\right)=(-1)^{n} \omega$, a contradiction when $n$ is odd.

Theorem 9. Assuming that the signature of the quadratic form is such that $p \geq 1$ and $q \geq 1$, the isometry $S_{p, p+1}=\sigma_{e_{p+1}} \sigma_{e_{p}}=\sigma_{e_{p}} \sigma_{e_{p+1}}$, is an involution, and it is an indirect isometry.

Proof. When $p=0$ or $q=0, S_{p, p+1}$ does not exist, so we assume that $p \geq 1$ and $q \geq 1$. From Theorem 3, $\sigma_{e_{p}} \sigma_{e_{p+1}}=\sigma_{e_{p+1}} \sigma_{e_{p}}$, and $S_{p, p+1}$ is involutive.

(a) Case where $p$ and $q$ are odd (thus, $p-1, q-1$, and $n$, are even). $\left(\sigma_{e_{2}} \sigma_{e_{1}}\right)$.

The full reflection is written: $\sigma_{\omega}=\left(\sigma_{e_{n}} \sigma_{e_{n-1}}\right) \ldots\left(\sigma_{e_{p+3}} \sigma_{e_{p+2}}\right) S_{p, p+1}\left(\sigma_{e_{p-1}} \sigma_{e_{p-2}}\right) \ldots$

In this product, the reflections are sequentially grouped into $n / 2$ pairs. Except $S_{p, p+1}$, these pairs of reflections generate rotations because their two supporting basis vectors 
have their squares with the same sign (see Theorem 6). It follows that $\sigma_{\omega}$ is a product of a rotation by $S_{p, p+1}$ and by another rotation. If $S_{p, p+1}$ would be a product of squares, $\sigma_{\omega}$ would also be a product of squares, a contradiction (Theorem 8 ), thus $S_{p, p+1}$ is an indirect isometry.

(b) Case where $p$ is odd and $q$ is even.

We consider $V$ as a subspace of a larger space, of odd dimensionality, with a quadratic form of signature $(p, q+1,0)$, so that there is one more unit basis vector of negative square, $e_{n+1}$. From part (a) of the proof, $S_{p, p+1}$ cannot be written as a product of squares. Thus, $S_{p, p+1}$ cannot be written as a product of squares in $V$ : it is an indirect isometry.

(c) Case where $p$ is even and $q$ is odd. The proof is similar to part (b), with a quadratic form of signature $(p+1, q, 0)$.

(d) Case where $p$ and $q$ are even. The proof is similar to parts (b) and (c), with a quadratic form of signature $(p+1, q+1,0)$.

Theorem 10. When $n$ is even:

(a) When $p$ and $q$ are even, $\sigma_{\omega}$ is a direct isometry.

(b) When $p$ and $q$ are odd, $\sigma_{\omega}$ is an indirect isometry.

Proof. It is recalled that the meaning of indirect isometries is the one of Definition 3.

(a) When $p$ and $q$ are even, $\sigma_{\omega}=\left(\sigma_{e_{n}} \sigma_{e_{n-1}}\right) \ldots\left(\sigma_{e_{p+2}} \sigma_{e_{p+1}}\right)\left(\sigma_{e_{p}} \sigma_{e_{p-1}}\right) \ldots\left(\sigma_{e_{2}} \sigma_{e_{1}}\right)$.

In this product, the reflections are sequentially grouped into $n / 2$ pairs, so that each of these pairs of reflections generates a rotation because their two supporting basis vectors have their squares with the same sign (see Theorem 6). It follows that $\sigma_{\omega}$ is a product of rotations, and as such it can be written as a product of squares.

(b) When $p$ and $q$ are odd, $n$ is even. As in part (a) of the proof of Theorem 9, we have $\sigma_{\omega}=\left(\sigma_{e_{n}} \sigma_{e_{n-1}}\right) \ldots\left(\sigma_{e_{p+3}} \sigma_{e_{p+2}}\right) S_{p, p+1}\left(\sigma_{e_{p-1}} \sigma_{e_{p-2}}\right) \ldots\left(\sigma_{e_{2}} \sigma_{e_{1}}\right)$, where $S_{p, p+1}$ is an indirect isometry and the $(n / 2)-1$ other pairs of reflections generate rotations. If $\sigma_{\omega}$ would be a product of squares, we could write $S_{p, p+1}$ as a product of squares, a contradiction. Therefore, $\sigma_{\omega}$ is an indirect isometry.

Definition 7. We define two reversal operators, $\sigma_{\omega_{+}}$and $\sigma_{\omega_{-}}$.

When $p \geq 1, \sigma_{\omega_{+}}=\sigma_{e_{p}} \sigma_{e_{p-1}} \ldots \sigma_{e_{1}}$. When $q \geq 1, \sigma_{\omega_{-}}=\sigma_{e_{n}} \sigma_{e_{n-1}} \ldots \sigma_{e_{p+1}}$.

Theorem 11. The following properties stand:

$\sigma_{\omega_{+}}$is an involution. It inverts the sign of the $p$ first components of any vector.

$\sigma_{\omega_{-}}$is an involution. It inverts the sign of the q last components of any vector.

The two reversal operators commute: $\sigma_{\omega_{-}} \sigma_{\omega_{+}}=\sigma_{\omega_{+}} \sigma_{\omega_{-}}=\sigma_{\omega}$.

Proof. Use Theorem 3 and Equation (3).

In the case where $\mathbb{R}^{1,3}$ is used to modelize the spacetime (mainly in special relativity), $\sigma_{\omega_{+}}$is the time reversal, and $\sigma_{\omega_{-}}$is the parity reversal.

In the case where $\mathbb{R}^{3,1}$ is used to modelize the spacetime (mainly in general relativity), $\sigma_{\omega_{+}}$is the parity reversal, and $\sigma_{\omega_{-}}$is the time reversal.

\subsection{Case of Non-Existence of a Square Root}

Theorem 12. Let $\sigma_{v} \sigma_{u}$ be the composition of the reflections $\sigma_{u}$ and $\sigma_{v}$, respectively associated with the invertible vectors $u$ and $v$. When $\operatorname{sgn}(u \cdot u) \neq \operatorname{sgn}(v \cdot v), \sigma_{v} \sigma_{u}$ is an indirect isometry, and it has no square root.

Proof. We assume that $p \geq 1$ and $q \geq 1$ (if not, all squared vectors have the same sign, $\sigma_{v} \sigma_{u}$ cannot exist). We remember that the indirect isometry $S_{p, p+1}=\sigma_{e_{p+1}} \sigma_{e_{p}}$ (also equal to $\sigma_{e_{p}} \sigma_{e_{p+1}}$ ), is an involution (Theorem 9). We observe that for any two invertible vectors $u$ and $v$ with their squares of opposite signs, the product of reflections $\sigma_{v} \sigma_{u}$ is an indirect isometry because, when $u^{2}>0$ and $v^{2}<0, \sigma_{v} \sigma_{u}=\left(\sigma_{v} \sigma_{e_{p+1}}\right)\left(\sigma_{e_{p+1}} \sigma_{e_{p}}\right)\left(\sigma_{e_{p}} \sigma_{u}\right)$, and when 
$u^{2}<0$ and $v^{2}>0, \sigma_{v} \sigma_{u}=\left(\sigma_{v} \sigma_{e_{p}}\right)\left(\sigma_{e_{p}} \sigma_{e_{p+1}}\right)\left(\sigma_{e_{p+1}} \sigma_{u}\right)$, which means in both cases that $\sigma_{v} \sigma_{u}$ is a product of a rotation by an indirect isometry and by a rotation, whence $\sigma_{v} \sigma_{u}$ is an indirect isometry.

It is not a product of squares, so it cannot be a square.

This non-existence of a square root is to compare with the case where $\operatorname{sgn}(u \cdot u)=$ $\operatorname{sgn}(v \cdot v)$, for which the square root exists (Theorem 6).

\subsection{Classification of Isometries}

This classification is based on Definition 3. We know that the identity is a direct isometry because it is the square of any reflection, and that a product of rotations is a direct isometry (see Corollary 4).

We also know that a single reflection is an indirect isometry (see Corollary 2), and that the full reflection is an indirect isometry, except when $p$ and $q$ are even, in which case the full reflection is a direct isometry (Theorems 8 and 10).

The full classification of the isometries of $O(p, q)$ is performed with Theorem 13, with the help of Lemma 6.

Lemma 6. $T$ being any isometry of $O(p, q), T$ and $S_{p, p+1} T S_{p, p+1}$ either are both direct isometries or are both indirect isometries.

Proof. We remember that $S_{p, p+1}$ is an involution (Theorem 9). We assume that $T$ is a direct isometry, so that it can be written: $T=T_{m}^{2} T_{m-1}^{2} \ldots T_{1}^{2}$, where $T_{k}, 1 \leq k \leq m$, are any isometries. If $S_{p, p+1} T S_{p, p+1}$ would be an indirect isometry, we would have $S_{p, p+1} T S_{p, p+1}=$ $\left(S_{p, p+1} T_{m} S_{p, p+1}\right)^{2}\left(S_{p, p+1} T_{m-1} S_{p, p+1}\right)^{2} \ldots\left(S_{p, p+1} T_{1} S_{p, p+1}\right)^{2}$, where the right side of this equality is a product of squares, a contradiction.

We assume that $T$ is an indirect isometry, so that it cannot be written as a product of squares. If $S_{p, p+1} T S_{p, p+1}$ could be written as a product of squares, i.e., $S_{p, p+1} T S_{p, p+1}=$ $T_{m}^{2} T_{m-1}^{2} \ldots T_{1}^{2}$, where $T_{k}, 1 \leq k \leq m$, are any isometries, we would have: $T=S_{p, p+1}$ $\left(S_{p, p+1} T S_{p, p+1}\right) S_{p, p+1}$, i.e.,:

$$
T=\left(S_{p, p+1} T_{m} S_{p, p+1}\right)^{2}\left(S_{p, p+1} T_{m-1} S_{p, p+1}\right)^{2} \ldots\left(S_{p, p+1} T_{1} S_{p, p+1}\right)^{2},
$$

where the right side of this equality is a product of squares, a contradiction.

Before proving Theorem 13, which classifies the isometries of $O(p, q)$, we recall that a product of direct isometries is a direct isometry, and that a product of a direct isometry by an indirect isometry (or conversely), is an indirect isometry. This is true in general, not only in quadratic spaces [9].

However, we recall that Definition 3 of direct and indirect isometries is not the classical one.

Theorem 13. A product of reflections is classified as direct or indirect as follows:

(a) When the product contains an odd number of reflections, it is an indirect isometry.

When the product contains an even number of reflections:

(b) It is a direct isometry when an even number of the squares of the supporting vectors have a negative sign, and the squares of the other supporting vectors have a positive sign.

(c) It is an indirect isometry when an odd number of the squares of the supporting vectors have a negative sign, and the squares of the other supporting vectors have a positive sign.

Proof. (a) We assume that the product of an odd number of reflections can be written as a product of squares. If so, one of these reflections can be decomposed into an even number of reflections, a contradiction (see Theorem 5).

- (b) and (c), case $p=0$ or $q=0$. All vectors have their squares with a common sign.

In case (b), a product of an even number of reflections is a direct isometry because it is a product of rotations (Corollary 4). 
In case (c), a product of an odd number of reflections is a product of a rotation by a single reflection, so it cannot be a direct isometry because a single reflection is an indirect isometry (Corollary 2).

- (b) and (c), case $p \geq 1$ and $q \geq 1$. We notice that given an isometry $T$, of direct or indirect nature, multiplying $T$ on its right or on its left by any rotation, gives another isometry, $T^{\prime}$, but $T$ and $T^{\prime}$ have the same direct or indirect nature because a rotation is a direct isometry.

We are in the case where $T$ contains an even number of reflections, so that their product can be partitioned into pairs of consecutive reflections. We consider a pair of consecutive reflections $\sigma_{u}$ and $\sigma_{v}$, supported respectively by the invertible vectors, $u$ and $v$.

When $u^{2}>0$ and $v^{2}<0, \sigma_{v} \sigma_{u}=\left(\sigma_{v} \sigma_{e_{p+1}}\right)\left(\sigma_{e_{p+1}} \sigma_{e_{p}}\right)\left(\sigma_{e_{p}} \sigma_{u}\right)$, and when $u^{2}<0$ and $v^{2}>0, \sigma_{v} \sigma_{u}=\left(\sigma_{v} \sigma_{e_{p}}\right)\left(\sigma_{e_{p}} \sigma_{e_{p+1}}\right)\left(\sigma_{e_{p+1}} \sigma_{u}\right)$, If there is no such pair, $T$ is a product of rotations, and case (b) of the theorem is proved. If there are such pairs, each one is an indirect isometry (see Theorem 12), and rewriting $T$ with replacement of each of them by a triplet of pairs as above, does not change the direct or indirect nature of $T$, because each triplet of pairs contains two rotations and one occurrence of the indirect isometry $S_{p, p+1}$ (see Theorem 9).

The expression of $T$ contains now only rotations and one or several occurrences of $S_{p, p+1}$. When two occurrences of $S_{p, p+1}$ are consecutive in $T$, their product is the identity, and it can be removed from $T$ (that does not change the direct or indirect nature of $T$ ). If no occurrence of $S_{p, p+1}$ remains, only rotations remain, and case (b) of the theorem is proved.

If it happens that the rightmost occurrence of $S_{p, p+1}$ in $T$ is followed on the right by a product of rotations, we multiply $T$ on its right by the inverse of this product of rotations, so that only the identity remains at the right of the rightmost occurrence of $S_{p, p+1}$. We perform similarly on the left, so that only the identity remains at the left of the leftmost occurrence of $S_{p, p+1}$. These identities are removed, and as noticed previously, it remains the expression of another isometry, $T^{\prime}$, so that $T$ and $T^{\prime}$ have the same direct or indirect nature.

At this step of the proof, $T^{\prime}$ has one occurrence of $S_{p, p+1}$ at its left and one at its right. If these latter two occurrences of $S_{p, p+1}$ coincide, i.e., only one occurrence of $S_{p, p+1}$ remains, $T^{\prime}$ and $T$ are indirect isometries, and case (c) is proved. We multiply $T^{\prime}$ on its left by $S_{p, p+1}$, and on its right by $S_{p, p+1}$. That changes neither the even or odd parity of the number of occurrences $S_{p, p+1}$, nor the direct or indirect nature of $T^{\prime}$ (see Lemma 6). Remembering that $S_{p, p+1}$ is involutive, we remove the identity on the left of $T^{\prime}$ and the identity on its right. As noticed previously, the rotations which stand now at the right and at the left of $T^{\prime}$ can be eliminated, and we retrieve again $S_{p, p+1}$ at the right and at the left of $T^{\prime}$. We return at the beginning of the current step of the proof, with several occurrences of $S_{p, p+1}$ decreased by two units. This step of the proof is repeated until it remains either one occurrence of $S_{p, p+1}$, which proves case (c), or none, which proves case (b).

\subsection{Comments on the Classification of Isometries}

When $p \geq 1$ and $q \geq 1$, the real orthogonal group $O(p, q)$ is a Lie group containing four connected components [17]. These components can be identified with the involutive operators defined in Section 4.3.

1. The component containing the identity, I.

2. The component containing the reversal operator $\sigma_{\omega_{+}}$.

3. The component containing the reversal operator $\sigma_{\omega_{-}}$.

4. The component containing the full reflection $\sigma_{\omega}=\sigma_{\omega_{+}} \sigma_{\omega_{-}}=\sigma_{\omega_{-}} \sigma_{\omega_{+}}$.

When $p=0$ or $q=0$, case (c) of Theorem 13 cannot be encountered. It means that the product of two indirect isometries is always a direct isometry. When $q=0$, as it happens in the Euclidean case, $O(p, q)$ contains only the components 1 and 2 . The component 1 is the direct subgroup (that is, the special orthogonal group $S O(n)$, or rotation group), and the component 2 contains the indirect isometries. When $p=0, O(p, q)$ contains only the components 1 and 3 .

In the case of $\mathbb{R}^{1,3}$, which is used to modelize the spacetime in special relativity, the Lorentz group is isomorphic to $O(1,3)$. The time reversal $T$ can be identified with $\sigma_{\omega_{+}}$, and 
the parity inversion $P$ can be identified $\sigma_{\omega_{-}}$. The components of the full Lorentz group are as follows.

1. The component 1 is the proper orthochronous Lorentz group.

2. The component 2 contains the antichronous proper Lorentz transformations.

3. The component 3 contains the orthochronous improper Lorentz transformations.

4. The component 4 contains the antichronous improper Lorentz transformations.

The even/odd paradigm which states that the product of two indirect isometries should be a direct isometry does not work anymore (Theorem 13). In the case of the Lorentz group, it means that $P T$ is classified as an indirect isometry (this result was proved in [16] using a matrix representation of the Lorentz group). This is a consequence of Definition 3.

The direct versus indirect classification based on Definition 3 may appear to be counterintuitive. To support that, we observe that $\left\{I, \sigma_{\omega_{+}}, \sigma_{\omega_{-}}, \sigma_{\omega}\right\}$, a discrete subrgoup of $O(p, q)$, is isomorphic to the Klein 4-group, as shown in Figure 1.

\begin{tabular}{c|cccc} 
& $I$ & $R$ & $G$ & $B$ \\
\hline$I$ & $I$ & $R$ & $G$ & $B$ \\
$R$ & $R$ & $I$ & $B$ & $G$ \\
$G$ & $G$ & $B$ & $I$ & $R$ \\
$B$ & $B$ & $G$ & $R$ & $I$
\end{tabular}

\begin{tabular}{c|cccc} 
& $I$ & $\sigma_{\omega_{+}}$ & $\sigma_{\omega_{-}}$ & $\sigma_{\omega}$ \\
\hline$I$ & $I$ & $\sigma_{\omega_{+}}$ & $\sigma_{\omega_{-}}$ & $\sigma_{\omega}$ \\
$\sigma_{\omega_{+}}$ & $\sigma_{\omega_{+}}$ & $I$ & $\sigma_{\omega}$ & $\sigma_{\omega_{-}}$ \\
$\sigma_{\omega_{-}}$ & $\sigma_{\omega_{-}}$ & $\sigma_{\omega}$ & $I$ & $\sigma_{\omega_{+}}$ \\
$\sigma_{\omega}$ & $\sigma_{\omega}$ & $\sigma_{\omega_{-}}$ & $\sigma_{\omega_{+}}$ & $I$
\end{tabular}

\begin{tabular}{c|cccc} 
& $I$ & $T$ & $P$ & $P T$ \\
\hline$I$ & $I$ & $T$ & $P$ & $P T$ \\
$T$ & $T$ & $I$ & $P T$ & $P$ \\
$P$ & $P$ & $P T$ & $I$ & $T$ \\
$P T$ & $P T$ & $P$ & $T$ & $I$
\end{tabular}

Figure 1. The Cayley table of the Klein 4-group. On the left: the general case, with three colors: $R$ : Red, G: Green, B: Blue. On the middle: case of the discrete subgroup of $O(p, q):\left\{I, \sigma_{\omega_{+}}, \sigma_{\omega_{-}}, \sigma_{\omega}\right\}$. On the right: case of the discrete subgroup of the Lorentz group: $\{I, T, P, P T\}$.

In Figure 1, the three colors $R, G, B$, play equivalent roles. There is no reason to classify $B$ (or $R$, or $G$ ), with $I$. Thus, it is more natural to classify $\sigma_{\omega_{+}}, \sigma_{\omega_{-}}$and $\sigma_{\omega}$ together, as indirect isometries, rather than to follow the even/odd paradigm, which intuitively leads to classify $\sigma_{\omega}$ (or $P T$ ) with $I$, as a direct isometry, as it happens in the Euclidean case.

Anyway, whichever terminology is used, the four components of $O(p, q)$ are known since a long time. However, the direct versus indirect definition of isometries presented in Section 4, works for any metric space, not only for quadratic spaces. This definition induced general rules of composition of isometries, except for the composition of indirect isometries [9]. These latter, presented in Section 4.5 (Theorem 13), are specific to quadratic spaces.

\section{Discussion}

Here, due to their importance, we must give a rough idea of how the terms chirality, right-handed and left-handed are used in modern physics. For a rigorous and pedagogical presentation, see [8]. Chirality is often related to the so-called $\gamma^{5}$ matrix, denoted here in contravariant notation. This matrix is generally defined as $\gamma^{5}= \pm i \gamma^{0} \gamma^{1} \gamma^{2} \gamma^{3}$, where $i$ is the imaginary number of square -1 , and where the four gamma matrices $\gamma^{0}, \gamma^{1}, \gamma^{2}$, and $\gamma^{3}$, generate a matrix representation of the Clifford algebra $C l_{1,3}(\mathbb{R})$. Here, we notice that in our presentation of geometric algebra, we did not encounter imaginary numbers. The gamma matrices satisfy to the anticommutation rules. Their squares are respectively $+1,-1,-1,-1$, using an abuse of notation for matrices that we retain in the rest of this paragraph. In the $(1 / 2,0) \oplus(0,1 / 2)$ representation of the Lorentz group, the gamma matrices have 4 lines and 4 columns, and act on Dirac bispinors, which are elements of $\mathbb{C}^{4}$. The Dirac bispinors goes back to 1928, when Dirac predicted the existence of the positron, using a four components vector, each component being a complex wavefunction (there are two wavefunctions for the electron, with spins $+1 / 2$ and $-1 / 2$, and two for the positron, with spins $+1 / 2$ and $-1 / 2$ ). However, at this time, neither spinors, nor Clifford algebra, and nor chirality were mentioned by Dirac. In a particular basis called the Weyl basis, the matrices $\left(1-\gamma^{5}\right) / 2$ and $\left(1+\gamma^{5}\right) / 2$ respectively projects Dirac bispinors to obtain their left-handed part (their two first components, associated with the eigenvalue -1 ) and their 
right-handed part (their two last components, associated with the eigenvalue +1 ). This appears to be the main explanation why $\gamma^{5}$ is called the chirality operator. To conclude about the physical concept of chirality, we can say that despite its mathematical foundations, it is unclear how to use it as a general definition of chirality.

Then, in 1952, Weyl explained in his book what are symmetry and chirality, their importance in nature, arts and science, and their strong relation with groups [18]. This book is sometimes cited as a reference for a definition of symmetry, but no unifying mathematical definition of symmetry and chirality was given there.

Finally, a dozen mathematical structures were named chiral in 2004 by Beilinson and Drinfeld [19], such as chiral algebra and chiral homology, without reference to older chirality concepts. Investigating the relations between these mathematical structures and Definition 4 of chirality, is out of the scope of this paper.

To conclude, as noticed in [20], there is a need for consensual mathematical definitions in the field of symmetry and chirality, in order to avoid ambiguities in the literature.

Funding: This research received no external funding.

Institutional Review Board Statement: Not applicable.

Informed Consent Statement: Not applicable.

Data Availability Statement: No data were generated during the study.

Conflicts of Interest: The author declares no conflict of interest.

\section{References}

1. Gal, J. Louis Pasteur, chemical linguist: founding the language of stereochemistry. Helv. Chim. Acta 2019, 102, e1900098.10.1002/hlca.201900098. [CrossRef]

2. Kelvin, W.T.B. The Molecular Tactics of a Crystal; Clarendon Press: Oxford, UK, 1894; Chapter 22, p. 27.

3. Kelvin, W.T.B. Baltimore Lectures on Molecular Dynamics and the Wave Theory of Light; C.J. Clay and Sons, Cambridge University Press Warehouse: London, UK, 1904; Chapter 22, Appendix H, Footnote, p. 619.

4. Eddington, A. Fundamental Theory; Cambridge University Press: Cambridge, UK, 1946; p. 111.

5. Hestenes, D.; Holt, J.W. Crystallographic space groups in geometric algebra. J. Math. Phys. 2007, 48, 023514.10.1063/1.2426416. [CrossRef]

6. Hitzer, E.; Perwass, C. Interactive 3D space group visualization with CLUCalc and the Clifford geometric algebra description of space group. Adv. Appl. Clifford Alg. 2010, 20, 631-658.10.1007/s00006-010-0214-z. [CrossRef]

7. Petitjean, M. A definition of symmetry. Symmetry Cult. Sci. 2007, 18, 99-119. Available online: https://hal.archives-ouvertes.fr/ hal-01552499 (accessed on 5 April 2021).

8. Schwichtenberg, J. Physics from Symmetry; Springer: Cham, Switzerland, 2018; Chapters 3.7, 6.3 and 7.4.

9. Petitjean, M. Chirality in metric spaces. In memoriam Michel Deza. Optim. Lett. 2020, 14, 329-338.10.1007/s11590-017-1189-7. [CrossRef]

10. Hestenes, D.; Sobczyk, G. Clifford Algebra to Geometric Calculus. A Unified Language for Mathematics and Physics; D. Reidel Publising Co.: Dordreht, The Netherlands, 1984; Chapters 1 and 3.8.

11. Macdonald, A. An elementary construction of the geometric algebra. Adv. Appl. Clifford Alg. 2002, 12, 1-6.10.1007/BF03161249. [CrossRef]

12. Doran, C.; Lasenby, A. Geometric Algebra for Physicists; Cambridge University Press: Cambridge, UK, 2013 ; Chapters 1, 2 and 4.

13. Aragón-González, G.; Aragón, J.; Rodríguez-Andrade, M. The decomposition of an orthogonal transformation as a product of reflections. J. Math. Phys. 2006, 47, 013509.10.1063/1.2161072. [CrossRef]

14. Artin, E. Geometric Algebra; Interscience: New York, NY, USA, 1957; Chapter III.4, pp. 129-131.

15. Lasenby, A. Geometric algebra as a unifying language for physics and engineering and its use in the study of gravity. Adv. Appl. Clifford Alg. 2017, 27, 733-759.10.1007/s00006-016-0700-z. [CrossRef]

16. Petitjean, M. About chirality in Minkowski spacetime. Symmetry 2019, 11, 1320.10.3390/sym11050662. [CrossRef]

17. Vaz, J.; Da Rocha, R., Jr. An Introduction to Clifford Algebras and Spinors; Oxford University Press: Oxford, UK, 2016; Chapter 5.1, p. 122.

18. Weyl, H. Symmetry; Princeton University Press: Princeton, NJ, USA, 1952.

19. Beilinson, A.; Drinfeld, V. Chiral Algebras; AMS Colloquium Publications: Providence, RI, USA, 2004 ; Chapter 3.

20. Petitjean M. Symmetry, antisymmetry and chirality: Use and misuse of terminology. Symmetry 2021, 13, 603.10.3390/sym13040603. [CrossRef] 